\title{
Mixofibroma lipoesclerosante de fémur proximal: controversias diagnósticas y terapéuticas. A propósito de 3 casos clínicos
}

\author{
Liposclerosing myxofibroma of the proximal femur: diagnostic and \\ therapeutic controversies. Apropos of 3 clinical cases \\ María Ángela Mellado Romero, ,; María Amparo Godoy Montijano, ${ }^{*}$ § Andrea Alcalá-Galiano Rubio,, , \\ Paula Casas Ramos, ${ }^{*}$, Ana Belén Enguita Valls, ${ }^{*, * *}$ Luis Rafael Ramos Pascua ${ }^{*, \neq}$ \\ *Servicio de Cirugía Ortopédica y Traumatología de la Unidad de Tumores Musculoesqueléticos; $¥$ Hospital Universitario 12 \\ de Octubre. Madrid, España; §Hospital Universitario Virgen de las Nieves. Granada, España; "Servicio de Radiología de la \\ Unidad de Tumores Musculoesqueléticos; "Hospital Universitario de León. León, España; **Servicio de Anatomía Patológica.
}

\begin{abstract}
Resumen
Introducción: El mixofibroma lipoesclerosante (MFLE) es un tumor benigno fibro-óseo poco frecuente con características clínico-radiológicas distintivas, si bien de diagnóstico y tratamiento controvertidos. Material y métodos: Se describen tres casos clínicos, dos mujeres y un hombre, con una edad media de 46 años, diagnosticados de MFLE. Se presentan los hallazgos de las pruebas complementarias de imagen y el tratamiento realizado: observación en dos pacientes y tratamiento quirúrgico (curetaje, relleno con homoinjerto óseo y fijación quirúrgica) en el tercero. Resultados: Los dos primeros pacientes, después de 10 y tres años de seguimiento, respectivamente, mantienen las mismas molestias y ausencia de progresión de la lesión. La paciente intervenida, con dos años de evolución, refiere dolor de menos intensidad al original sin signos de recidiva tumoral. Conclusiones: El diagnóstico de MFLE puede asumirse por datos clínicos y de imagen sin necesidad de biopsia. En ausencia de signos que hagan sospechar malignidad está justificada la vigilancia estrecha, limitándose la indicación quirúrgica a los casos sintomáticos y/o con riesgo de fractura patológica.
\end{abstract}

Palabras clave: Mixofibroma lipoesclerosante, tumor óseo, cadera, malignización.

\section{Abstract}

Introduction: The bone liposclerosing myxofibrous tumour (LSMFT) is a rare benign fibro-osseous tumor with characteristic clinicalradiological features, although its diagnosis and treatment are controversial. Material and methods: We present three patients, two women and one man, with a mean age of 46 years, diagnosed with LSMFT. The findings of the complementary imaging tests and the treatment performed are presented: observation in two patients and curettage, and the third patient was treated with curettage, grafting and surgical fixation. Results: The first two patients, after 10 and three years of follow-up, respectively, maintain the same symptoms and no progression of the lesion. Conclusions: The diagnosis of LSMFT can be assumed by clinical and imaging data without biopsy. In the absence of signs that suggest malignancy, observation is justified, limiting the surgical indication to symptomatic cases and/or those with risk of pathological fracture.

Keywords: Liposclerosing myxofibrous tumor, bone tumor, hip, malignizant transformation.

\section{Introducción}

El mixofibroma lipoesclerosante (MFLE), descrito inicialmente por Ragsdale y Sweet ${ }^{1}$ en 1986, es un tumor benigno fibro-óseo con características clínico- radiológicas distintivas y patrones histológicos diversos..$^{2,3}$ La lesión suele diagnosticarse en la cuarta década de la vida, sin predominio de un sexo sobre otro, ${ }^{4}$ a menudo de forma incidental. A veces también con motivo de dolor intenso o de una fractura pato-

Correspondencia:

María Amparo Godoy Montijano

E-mail: amparogodoy@ hotmail.com

Recibido: 15-11-2021. Aceptado: 21-11-2021.

Citar como: Mellado RMÁ, Godoy MMA, Alcalá-Galiano RA, Casas RP, Enguita VAB, Ramos PLR. Mixofibroma lipoesclerosante de fémur proximal: controversias diagnósticas y terapéuticas. A propósito de 3 casos clínicos. Orthotips. 2022; 18 (1): 41-48. https://dx.doi.org/10.35366/103731 
lógica. ${ }^{4}$ De los casos, $85 \%$ se localizan en el fémur proximal, sobre todo en la región intertrocantérea, aunque este asiento no es exclusivo. ${ }^{2}$ Existen casos descritos en ilion, húmero, tibia, cráneo, costilla y acetábulo..$^{5-9}$ Desde el punto de vista radiológico se trata de una lesión lítica, con patrón geográfico, de margen bien definido y por lo regular esclerótico; con frecuencia con mineralización en su interior o un cierto grado de expansión en el contorno.6,10,11

Microscópicamente el tumor puede presentar una gran variedad de patrones: zonas de lipoma, de fibroxantoma, mixoma, mixofibroma, tejido fibroso, formaciones quísticas, actividad osteoclástica, trabéculas óseas similares a la displasia fibrosa, necrosis grasa, tejido óseo isquémico y rara vez, componentes cartilaginosos, ${ }^{5,12}$ todo ello en proporciones variables. ${ }^{6}$ Se incluye, pues, en el diagnóstico diferencial de las lesiones fibro-óseas, particularmente en la región trocantérea del fémur. Por otra parte, muestra un potencial riesgo de transformación maligna que se calcula entre 10 y $16 \%$ de los casos. ${ }^{5,6,9}$ Por este motivo es controvertida la necesidad de una biopsia para asegurar el diagnóstico y el tratamiento de la lesión. En concreto, si la observación expectante es suficiente o se precisa un tratamiento quirúrgico. El objetivo del presente estudio es discutir sobre estos aspectos con base en la bibliografía actual y nuestra experiencia de tres casos clínicos.

\section{Material y métodos}

En las unidades de tumores musculoesqueléticos de los hospitales universitarios de León y 12 de Octubre de Madrid, entre los años 2010 y 2021, se han revisado tres pacientes con diagnóstico de MFLE (Tabla 1).

Caso 1. Paciente varón de 47 años de edad sin antecedentes de interés, remitido para valoración de una imagen osteolítica en la región trocantérea del fémur izquierdo descubierta con motivo de un episodio transitorio de dolor cinco meses antes en la cadera, sin antecedente traumático (Figura 1A). El dolor fue de intensidad leve-moderada y desapareció espontáneamente, manteniéndose en la actualidad unas molestias muy discretas. La exploración física no reveló signos de interés. La TC (tomografía computarizada) y la RM (resonancia magnética) se muestran en las Figuras 2 y 3 . Se realizó seguimiento clínico y radiográfico trimestral durante dos años, semestral hasta el quinto y anual hasta la actualidad.

Caso 2. Mujer de 47 años de edad remitida desde otro centro con sospecha de lesión condral en el fémur proximal. La paciente refería molestias ocasionales en su cadera derecha de varios meses de evolución sin antecedente traumático (Figura 4). Discutido el caso en nuestro comité, se concluyó con el diagnóstico de sospecha de MFLE. Puesto que la sintomatología era ocasional y no limitante, se convino en un tratamiento conservador con vigilancia clínico-radiológica trimestral en los primeros dos años y semestral en el siguiente.

Caso 3. Mujer de 45 años de edad a la que, con motivo de un dolor abdominal bajo irradiado a su ingle derecha de gran intensidad, su ginecólogo le realizó una RM de pelvis, donde se descubrió la lesión ósea que motivó la derivación a nuestra unidad. En el estudio mediante TC se observó una lesión lítica expansiva de localización metafiso-diafisaria de $3 \times$ $4 \times 6.4 \mathrm{~cm}$ ubicada en cuello femoral derecho, con patrón geográfico de márgenes bien definidos y borde escleroso completo. No se observó discontinuidad cortical ni reacción perióstica. Completado el estudio de imágenes (Figuras 5 y 6), se diagnosticó MFLE del fémur proximal derecho. Debido al tamaño de la lesión (extendida y que ocupa la mayor parte del cuello femoral) y a la intensa sintomatología álgica, se decidió tratamiento quirúrgico. Bajo anestesia intradural en mesa ortopédica se realizó curetaje bajo

Tabla 1: Resumen de los casos de la serie.

\begin{tabular}{ccccccc}
\hline Caso & Edad (años) & Sexo & Clínica & Tratamiento & Seguimiento (años) & Resultados \\
\hline 1 & 47 & Hombre & Molestias no limitantes & Observación & 10 & $\begin{array}{c}\text { Mismas molestias } \\
\text { No cambios en imagen } \\
\text { Mismas molestias } \\
\text { No cambios en imagen } \\
\text { Dolor (EVA 5/10) } \\
\text { No recidiva }\end{array}$ \\
3 & 47 & Mujer & $\begin{array}{c}\text { Molestias ocasionales } \\
\text { no limitantes } \\
\text { Dolor (EVA 10/10) }\end{array}$ & Observación & 3 & $\begin{array}{c}\text { Curetaje, homoinjerto } \\
\text { y tornillo placa }\end{array}$ \\
\hline
\end{tabular}

$E V A=$ escala visual analógica . 



Figura 2:

Caso 1. A) Imagen coronal de tomografía computarizada.

B y C) Imágenes axiales a diferentes alturas.

control radioscópico y relleno de la cavidad resultante con homoinjerto estructural y triturado a compresión, además de estabilización con una placa con tornillo deslizante tipo AMBI (Smith-Nephew) (Figura 7). El aspecto macroscópico y microscópico de la lesión se observa en la Figura 8. En los cortes histológicos se reconocía tejido óseo maduro lamelar sin atipia entremezclado con áreas de aspecto xantomizado y otras en las que se depositaba hemosiderina. Además, se identificaban otras que recordaban la displasia fibrosa, con trabéculas de aspecto curvilíneo de gran tamaño, sin ribete osteoblástico, sobre un estroma celular fibrótico en el que las células presentaban un aspecto fibroblástico sin atipia citológica ni figuras de mitosis. En otras áreas el fondo era laxo, con focalidad de diferenciación mixoide y celularidad estrellada de 
prolongaciones mal delimitadas. Con todo se emitió el informe de MFLE.

\section{Resultados}

Los dos primeros pacientes, después de 10 y tres años de seguimiento, respectivamente, continúan con molestias locales inespecíficas que no les limita la actividad. En la actualidad realizan una vida rigurosamente normal. Las pruebas complementarias de imagen no han advertido cambios con respecto a las originales ni muestran signos de agresividad (Figuras
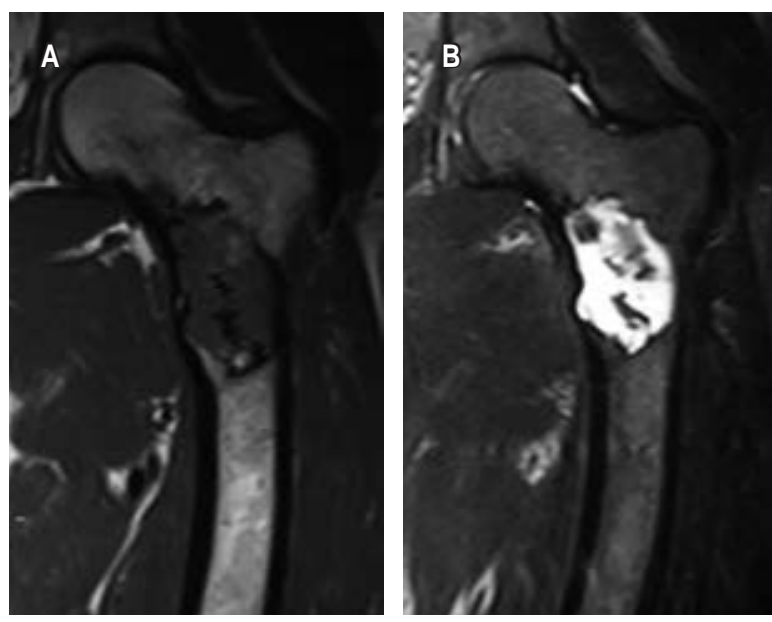

Figura 3: Caso 1. A) Imagen coronal de resonancia magnética en secuencias ponderadas en T1. B) Densidad protónica con saturación de la grasa (PDFS, por sus siglas en inglés).
1B-C y 9). En la tercera paciente no hubo complicaciones intraoperatorias ni postoperatorias. Después de dos años de seguimiento refiere persistencia del dolor, si bien de menor intensidad que antes de la intervención. En el control radiográfico se observan signos de consolidación del injerto (Figura 10) y dos años después de la cirugía no existe recidiva de la enfermedad.

\section{Discusión}

El tumor MFLE es un tumor benigno muy raro de etiopatogenia desconocida que podría malignizarse, quizás en relación con cambios involutivos e isquémicos intralesionales. ${ }^{9,13} \mathrm{Si}$ bien se han publicado series de $95,{ }^{5} 39^{6}$ y $33^{10}$ pacientes, la mayoría son de casos clínicos aislados. A pesar de su complejidad histológica, con los datos epidemiológicos y los característicos hallazgos de imagen, el diagnóstico se puede hacer sin biopsia en los casos típicos. ${ }^{10,11}$ No obstante, cuando se dude del diagnóstico, máxime si no se descarta malignidad, la biopsia es obligada. De cualquier forma, el uso del término y la enfermedad en general puede prestarse a confusión y motivar tratamientos inadecuados. ${ }^{10}$

El diagnóstico diferencial del MFLE debe incluir la displasia fibrosa monostótica y el lipoma intraóseo, con los que se ha relacionado sugiriéndose que fuera una forma de la primera o un estadio degenerativo del segundo. ${ }^{9,10}$ La displasia fibrosa en RM presenta menos esclerosis y por lo regular intensidad de señal baja o moderada en secuencias ponderadas en T2. ${ }^{11}$
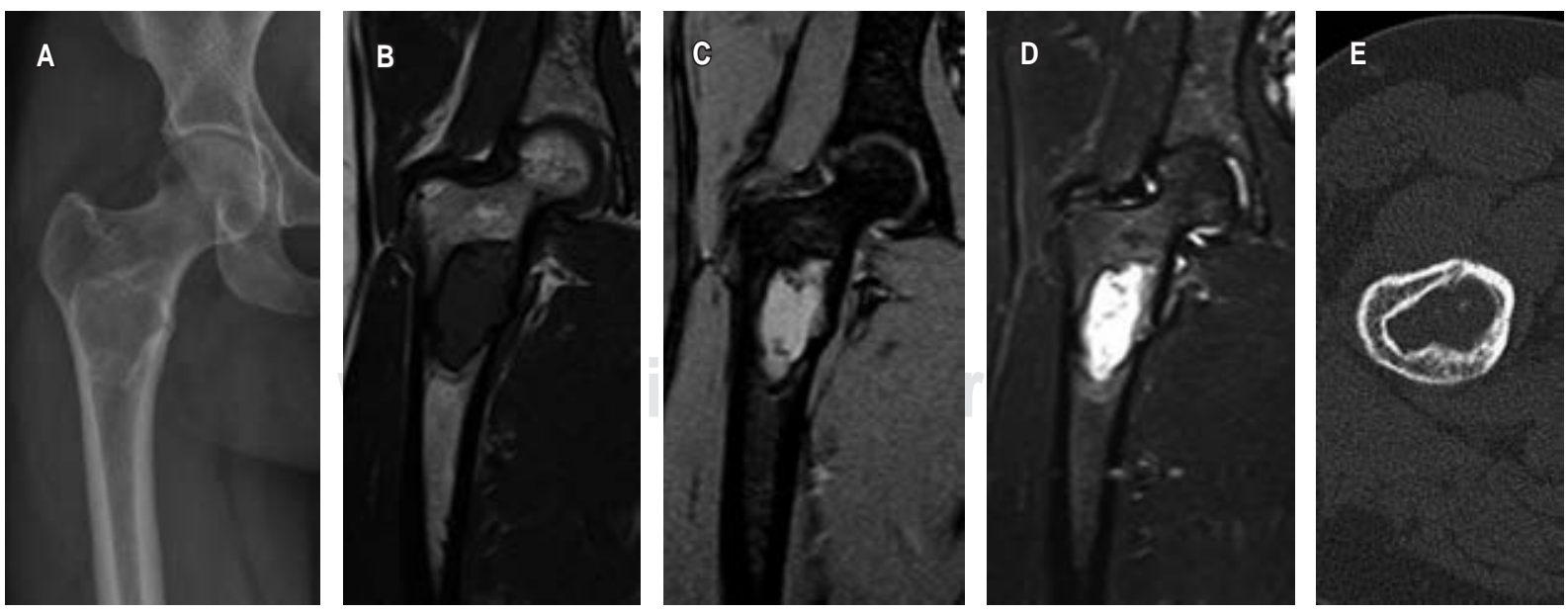

Figura 4: Caso 2. A) Radiografía en proyección anteroposterior. B-D) Imágenes coronales de resonancia magnética en secuencias T1, fast field echo y densidad protónica con saturación de la grasa (PDFS, por sus siglas en inglés). E) Imagen axial de tomografía computarizada. 

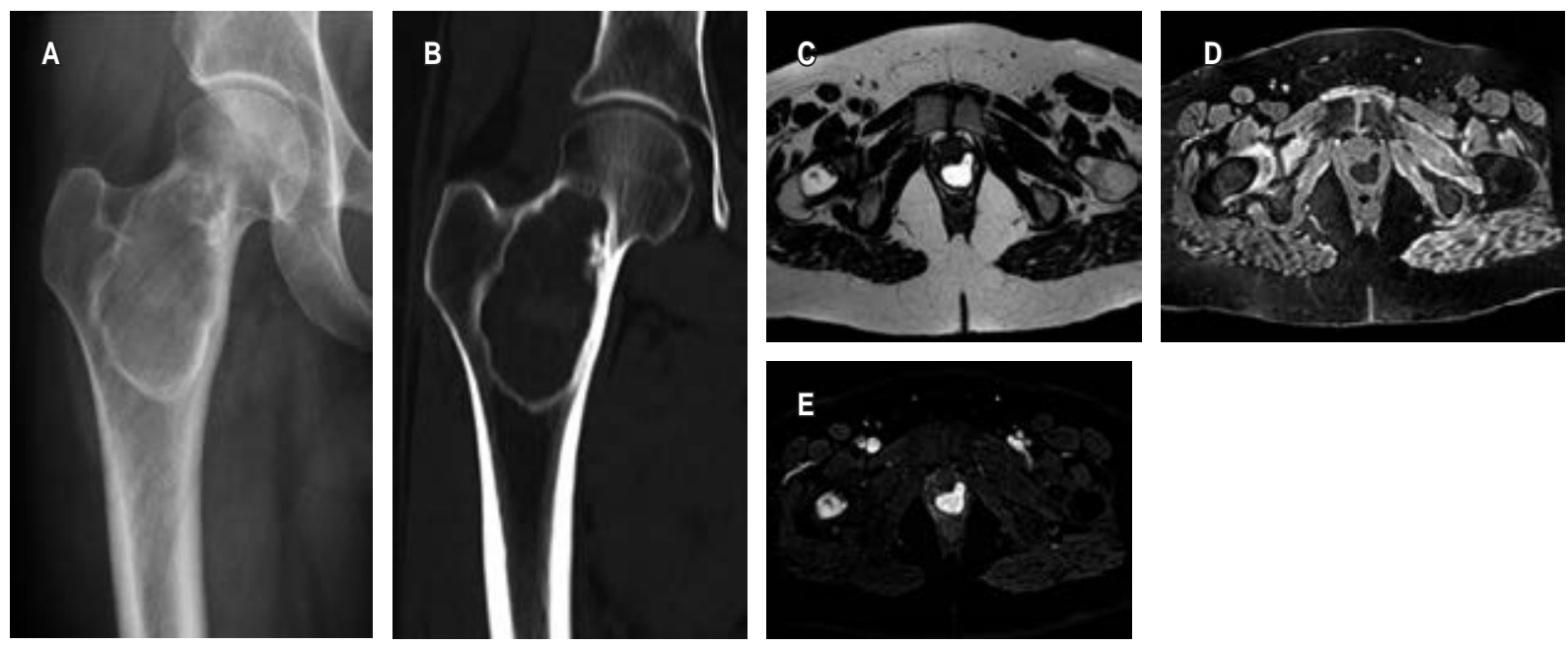

Figura 5: Caso 3. A) Radiografía anteroposterior. B) Imagen coronal de tomografía computarizada. C-E) Imágenes axiales de resonancia magnética en secuencias $\mathrm{T} 2, \mathrm{~T} 1$ con saturación de la grasa y eco de gradiente.

Desde el punto de vista histológico pueden ser indistinguibles morfológicamente y a veces, sólo se pueden diferenciar por estudio genético. ${ }^{14}$ El lipoma intraóseo es un tumor benigno centromedular de localización por lo general metafisaria en los huesos largos de los miembros, aunque también relativamente frecuente en el calcáneo, ilion y costillas. Suele diagnosticarse en la cuarta y quinta décadas de la vida, aunque el rango etario es amplio y puede cursar con dolor. El aspecto radiográfico del lipoma intraóseo depende del grado de involución y necrosis, con componente variable de grasa intralesional, degeneración quística y calcificaciones. El MFLE no suele presentar grasa macroscópica, ya que el componente lipomatoso es pequeño y se encuentra entremezclado con los componentes mixofibrosos o fibro-óseos. Otras lesiones a tener en cuenta en el diagnóstico diferencial radiológico serían el infarto óseo, el encondroma, el quiste óseo, el fibroma no osificante y el condrosarcoma. ${ }^{8,10}$ En nuestra serie, el diagnóstico de los casos se estableció con las pruebas clínicas y de imagen, ratificándose en el que intervenimos, lo cual sirvió para la descripción de su histopatología y la consideración de las lesiones como entidad propia independiente.

En la actualidad, el tratamiento del MFLE es controvertido. Los patrones histológicos más comunes en el MFLE malignizado incluyen el de osteosarcoma, histiocitoma fibroso maligno y tumores de células fusiformes de alto grado. ${ }^{5,6,13} \mathrm{Si}$ el riesgo de malignización realmente fuera de $10-16 \%$ de los casos, como algunos han descrito, $, 5,6,9$ se podría con-


Figura 6:

Caso 3. Gammagrafía ósea con Tc99 con mínima captación local de la lesión.

templar la resección en todos los escenarios. Si ese riesgo estuviera sobredimensionado, como parece deducirse de la revisión del conjunto de la literatura científica, ${ }^{10,13,15}$ el tratamiento quirúrgico podría ser innecesario en bastantes ocasiones. También se cuestionaría el tratamiento quirúrgico teniendo en cuenta que algún osteosarcoma o histiocitoma fibroso maligno identificado en el contexto de un MFLE podría haber surgido de novo o en el contexto de 

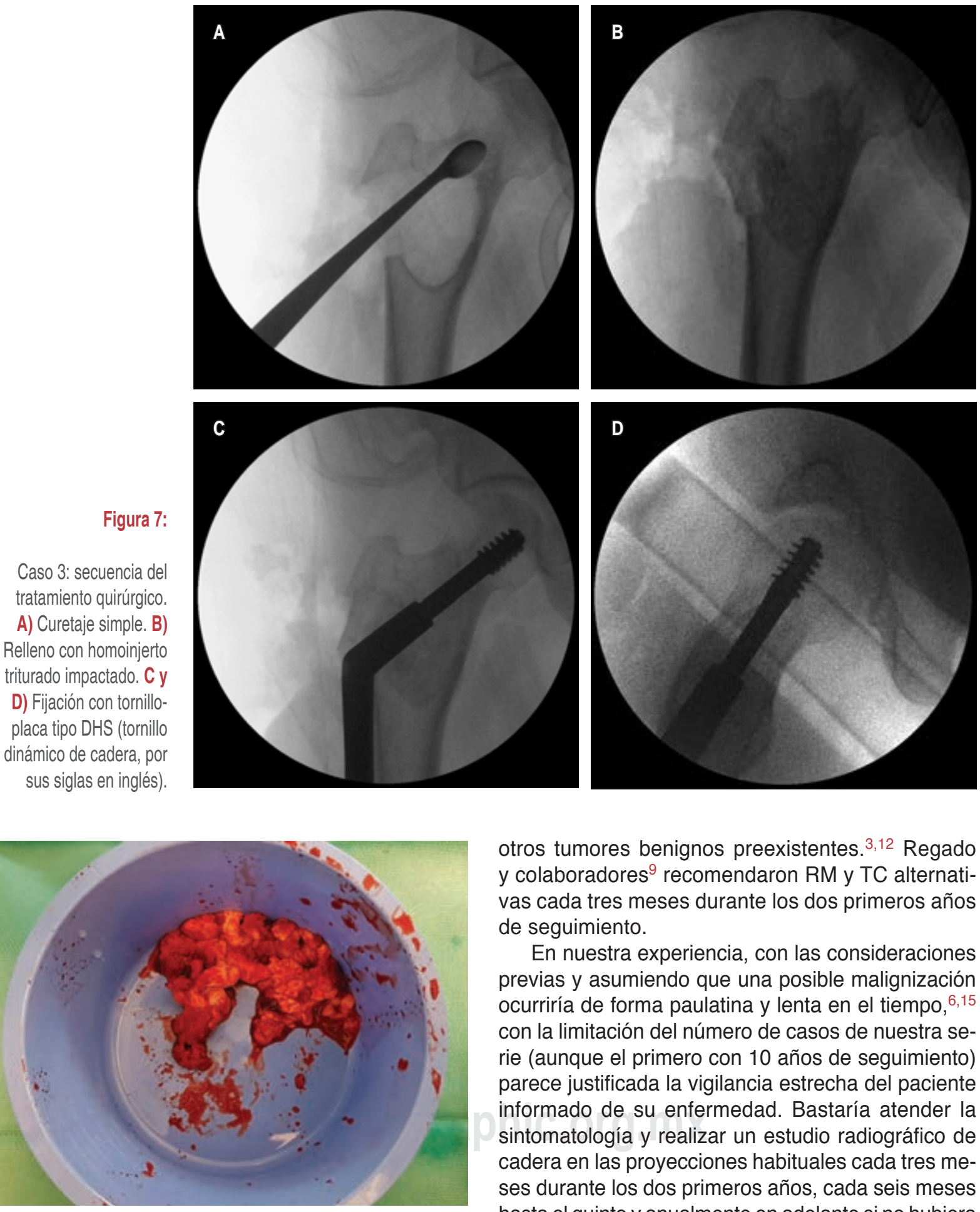

Figura 8: Caso 3. A) Aspecto macroscópico del material curetado. B) Hematoxilina-eosina 4x: trabéculas de hueso neoformado, curvilíneo, sobre fondo de aspecto fibroso. C) Hematoxilina-eosina 10x: áreas predominantemente fibróticas con zonas de xantomización (*).

otros tumores benignos preexistentes. ${ }^{3,12}$ Regado y colaboradores ${ }^{9}$ recomendaron RM y TC alternativas cada tres meses durante los dos primeros años de seguimiento.

En nuestra experiencia, con las consideraciones previas y asumiendo que una posible malignización ocurriría de forma paulatina y lenta en el tiempo,, 15 con la limitación del número de casos de nuestra serie (aunque el primero con 10 años de seguimiento) parece justificada la vigilancia estrecha del paciente informado de su enfermedad. Bastaría atender la sintomatología y realizar un estudio radiográfico de cadera en las proyecciones habituales cada tres meses durante los dos primeros años, cada seis meses hasta el quinto y anualmente en adelante si no hubiera modificaciones clínicas ni de imagen. Si las hubiera, completaríamos el estudio con TC y RM e indicaríamos una biopsia antes del tratamiento. Si se confirmara la malignidad realizaríamos una resección en 


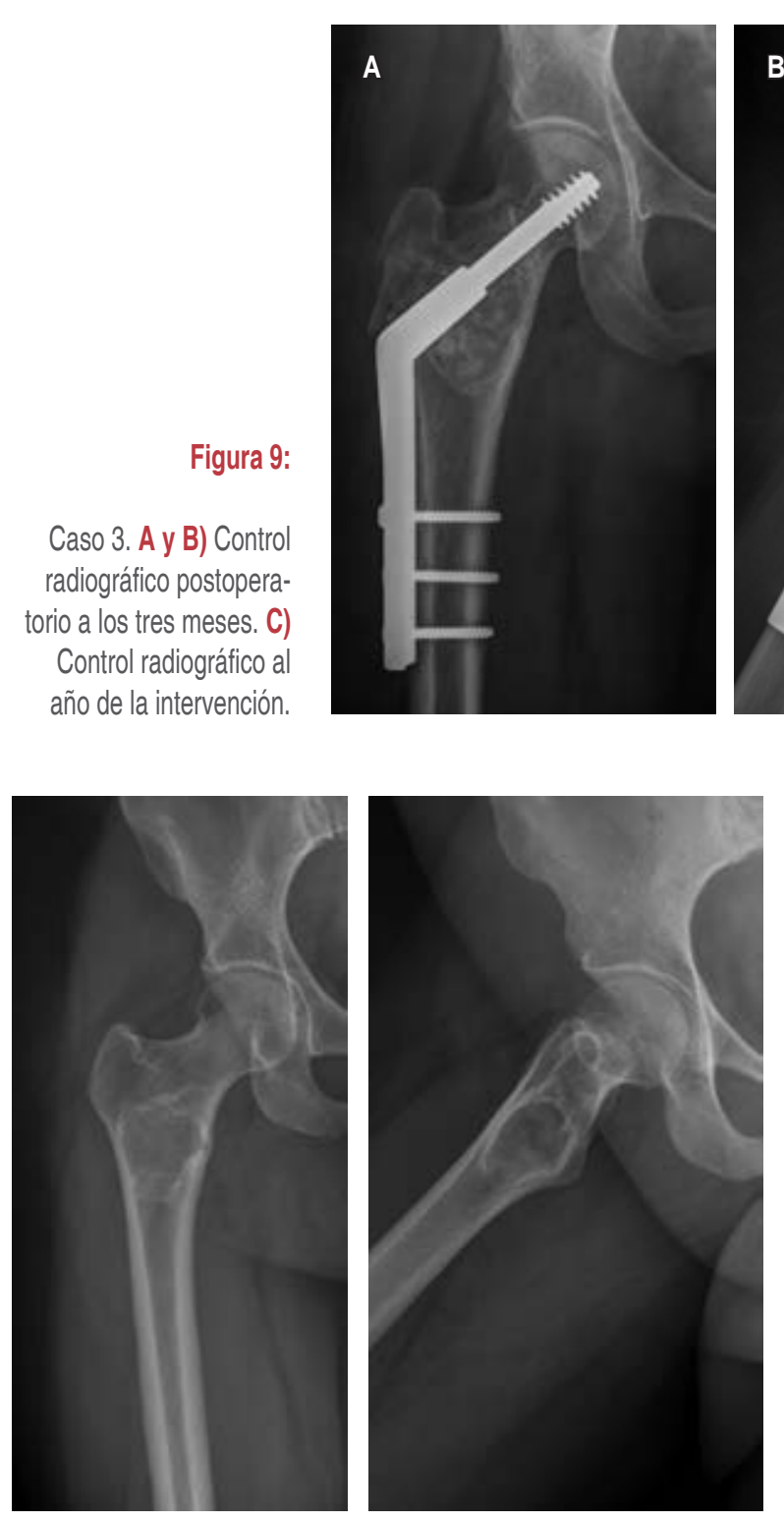

Figura 10: Caso 2. Control radiográfico anteroposterior y axial tres años después del diagnóstico.

bloque con márgenes amplios y reconstrucción protésica, habiéndose descrito hasta amputaciones. ${ }^{9,15}$ Si no sospecháramos de malignización indicaríamos el tratamiento quirúrgico en el supuesto de estimar un alto riesgo de fractura patológica en algún momento de la evolución de la enfermedad, como fue en nuestro caso 3. La técnica quirúrgica sería una resección intralesional con la opción de emplear algún tipo de terapia adyuvante seguido del relleno del defecto y algún tipo de fijación. ${ }^{2,4}$
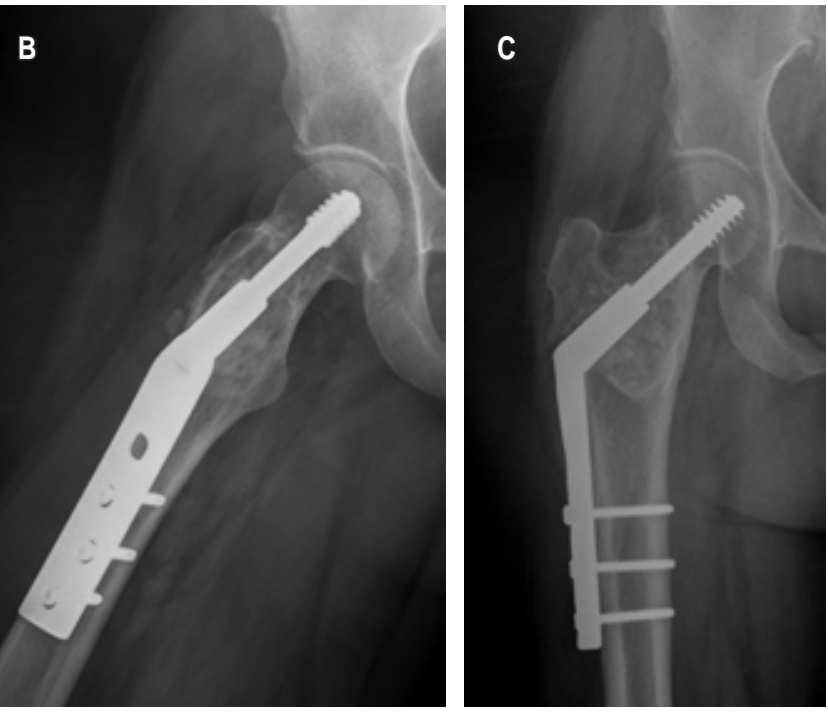

\section{Conclusiones}

El diagnóstico de MFLE puede asumirse por datos clínicos y de imagen sin biopsia. En ausencia de signos que sugieran malignidad está justificada la observación, limitando la indicación quirúrgica a casos sintomáticos y/o con riesgo de fractura patológica.

\section{Referencias}

1. Ragsdale BD, Sweet DE. Bone. In: Henson E, AlboresSaavedra J, eds. Pathology of incipient neoplasia. Philadelphia: WB Saunders; 1986. pp. 381-423.

2. Beytemür O, Tetikkurt US, Albay C, Kavsut G, Gülec A. Liposclerosing myxofibrous tumor: a rare tumor of proximal femur. Eklem Hast Ve Cerrahisi Jt Dis Relat Surg. 2017; 28 (3): 210-213. doi: 10.5606/ehc.2017.48394

3. Gilkey FW. Liposclerosing myxofibrous tumor of bone. Hum Pathol. 1993; 24: 1264. doi: 10.1016/0046-8177(93)90226-7.

4. Deel C, Hassell L. Liposclerosing myxofibrous tumor: a review. Arch Pathol Lab Med. 2016; 140: 473-476. doi: 10.5858/20140503-RS.

5. Ragsdale BD. Polymorphic fibro-osseous lesions of bone: an almost site-specific diagnostic problem of the proximal femur. Hum Pathol. 1993; 24 (5): 505-512. doi: 10.1016/00468177(93)90162-a.

6. Kransdorf MJ, Murphey MD, Sweet DE. Liposclerosing myxofibrous tumor: a radiologic-pathologic-distinct fibroosseous lesion of bone with a marked predilection for the intertrochanteric region of the femur. Radiology 1999; 212 (3): 693-698. doi: 10.1148/radiology.212.3.r99se40693.

7. Matsuba A, Ogose A, Tokunaga K, Kawashima H, Hotta T, Urakawa S, et al. Activating Gs alpha mutation at the Arg201 codon in liposclerosing myxofibrous tumor. Hum Pathol. 2003; 34: 1204-1209. doi: 10.1016/s0046-8177(03)00430-1.

8. Técualt-Gómez R, Atencio-Chan A, Cario-Méndez AG, Amaya-Zepeda RA, Balderas-Martinez J, GonzálezValladares JR. Bone liposclerosing myxofibrous tumor. Case 
presentation and literature review. Acta Ortop Mex. 2015; 29 (3): 191-195.

9. Regado ER, Garcia PB, Caruso AC, de Almeida AL, Aymoré IL, Meohas W, et al. Liposclerosing myxofibrous tumor: a series of 9 cases and review of the literature. J Orthop. 2016; 13: 136-139. doi: 10.1016/j.jor.2016.03.003.

10. Dattilo J, McCarthy EF. Liposclerosing myxofibrous tumor (LSMFT), a study of 33 patients: should it be a distinct entity? lowa Orthop J. 2012; 32: 35-39.

11. Nieto A, Pérez-Andrés R, Lorenzo JC, Vilanova JC. Diagnostic imaging of liposclerosing myxofibrous tumor of bone. Radiologia. 2010; 52 (3): 251-254. doi: 10.1016/j.rx.2009.12.012.

12. Heim-Hall JM, Williams RP. Liposclerosing myxofibrous tumour: a traumatized variant of fibrous dysplasia? Report of four cases and review of the literature. Histopathology. 2004; 45: 369-376. doi: 10.1111/j.1365-2559.2004.01951.x.

13. Murphey MD, Carroll JF, Flemming DJ, Pope TL, Gannon $\mathrm{FH}$, Kransdorf MJ. From the archives of the AFIP: benign musculoskeletal lipomatous lesions. Radiogr Rev Publ Radiol Soc N Am Inc. 2004; 24: 1433-1466. doi: 10.1148/ rg.245045120.

14. Corsi A, De Maio F, Ippolito E, Cherman N, Gehron Robey P, Riminucci M, et al. Monostotic fibrous dysplasia of the proximal femur and liposclerosing myxofibrous tumor: which one is which? J Bone Miner Res. 2006; 21: 1955-1958. doi: 10.1359/ jbmr.060818.

15. Campbell K, Wodajo F. Case report: two-step malignant transformation of a liposclerosing myxofibrous tumor of bone. Clin Orthop. 2008; 466: 2873-2877. doi: 10.1007/s11999-0080362-9.

\section{Conflicto de intereses}

Los autores no tienen conflicto de intereses en relación con este manuscrito. 